\title{
The Role of an Amygdalo-Nigrostriatal Pathway in Associative Learning
}

\author{
Jung-Soo Han, ${ }^{1}$ Robert W. McMahan, ${ }^{1}$ Peter Holland, ${ }^{2}$ and Michela Gallagher ${ }^{1}$ \\ ${ }^{1}$ Department of Psychology, University of North Carolina at Chapel Hill, Chapel Hill, North Carolina 27599, and \\ 2Department of Psychology, Duke University, Durham, North Carolina 27706
}

The present study examined the role of an amygdalonigrostriatal pathway in associative learning. An asymmetrical lesion model was used to test whether a circuit from the amygdala central nucleus to the dorsolateral striatum, via the substantia nigra, is critical for mediating conditioned orienting responses. Rats with an asymmetrical lesion, consisting of neurotoxic removal of central nucleus neurons in one hemisphere and depletion of the dopamine innervation of the dorsolateral striatum in the contralateral hemisphere, failed to acquire conditioned orienting responses. In contrast, the asymmetrical lesion had no effect on spontaneous orienting or learning another response directed to the source of the food unconditioned stimulus in the same task. A second experiment tested the effect of reversible inactivation of the dorsolateral striatum contralateral to a neurotoxic central nucleus lesion on acquisition of the conditioned orienting response. Although inactivation did not affect spontaneous orienting, rats failed to acquire the conditioned orienting response during sessions in which inactivation occurred. Immediately after the inactivation procedure was terminated, however, a significant increase in orienting to the conditioned stimulus was evident. These data support the interpretation that the dorsolateral striatum provides a route for the expression of the conditioned orienting response but is not essential for acquisition of this learned behavior.

Key words: learning and memory; pavlovian conditioning; conditioned orienting behavior; amygdala; striatum; asymmetrical lesion; ibotenic acid; lidocaine 6-OHDA
The amygdala central nucleus $(\mathrm{CN})$ is important for the modulation of orienting/attentional responses to signals for biologically significant events (Gallagher and Holland, 1994). In an appetitive conditioning procedure that permits evaluation of two types of learned behavior, normal rats acquire conditioned orienting responses to cues (stimuli such as lights or tones) that signal food delivery and learn to approach the site where food will be delivered (Holland, 1984). Rats with bilateral neurotoxic lesions of CN are unaffected in acquisition of behavior directed to the food delivery site but fail to acquire conditioned orienting responses to cues in both visual and auditory modalities (Gallagher et al., 1990). Notably, those same lesions have no effect on spontaneous orienting to cues presented as novel stimuli before conditioning (Sananes and Campbell, 1989; Gallagher et al., 1990).

Damage to the dorsolateral striatum in rats is known to impair orienting to sensory cues in rats. Unilateral damage to the nigrostriatal dopamine (DA) system (at its origin or in the striatum) produces a deficit in orienting to stimuli presented contralateral to the site of the lesion (Ljungberg and Ungerstedt, 1976). This deficit has been described as an inability to integrate sensory information with the performance of the orienting response, socalled intentional neglect (Carli et al., 1985), and depends on lateral but not medial striatum (Carli et al., 1989; Fairley and Marshall, 1989).

The present study examined the possibility that orienting re-

Received Jan. 7, 1997; revised Feb. 24, 1997; accepted Feb. 27, 1997.

This research was supported by grants from a Research Scientist Award (KO5MH01149), the Human Frontier Science Program, and Grant RO1-MH53667 to P.C.H. and M.G.

Correspondence should be addressed to Michela Gallagher, Department of Psychology, Davie Hall, CB\#3270, The University of North Carolina, Chapel Hill, NC 27599.

Copyright (C) 1997 Society for Neuroscience 0270-6474/97/173913-07\$05.00/0 sponses based on associative learning critically depend on an amygdalo-nigrostriatal pathway. The amygdala $\mathrm{CN}$ projects to many targets in the midbrain and brainstem; some of these targets provide direct somatomotor or autonomic output, whereas others provide widespread ascending innervation of forebrain systems. Among its targets, the amygdala $\mathrm{CN}$ has a substantial projection to a division of the substantia nigra compacta (SNC) that provides dopaminergic innervation of the dorsolateral striatum (Gonzales and Chesselet, 1990). Because the $\mathrm{CN}$ projection to $\mathrm{SNC}$ is ipsilateral and the output from SNC to the dorsolateral striatum (ST) is also largely ipsilateral, the function of this circuitry in conditioned orienting was examined using an asymmetrical lesion, illustrated in the schematic shown in Figure 1. This preparation, which combines a lesion of amygdala $\mathrm{CN}$ on one side of the brain (unilateral) with a contralateral removal of the dopaminergic innervation of the dorsolateral striatum, tests whether the amygdala-striatal pathway is critical for conditioned orienting. In a second study, the dorsolateral striatum was transiently inactivated during conditioning sessions to determine whether only the expression of conditioned responses was prevented by this reversible lesion. This was tested by monitoring the appearance of conditioned orienting responses immediately after cessation of the inactivation procedure. Both studies support a role for an amygdalo-striatal pathway in this simple form of associative learning, and further indicate that intact function of the dorsolateral striatum is not essential for acquisition of the conditioned response.

\section{Experiment 1}

The asymmetrical lesion preparation is illustrated in Figure 1. Because a $\mathrm{CN}$ projection to the subset of DA neurons that innervate the dorsolateral striatum is confined to the ipsilateral 


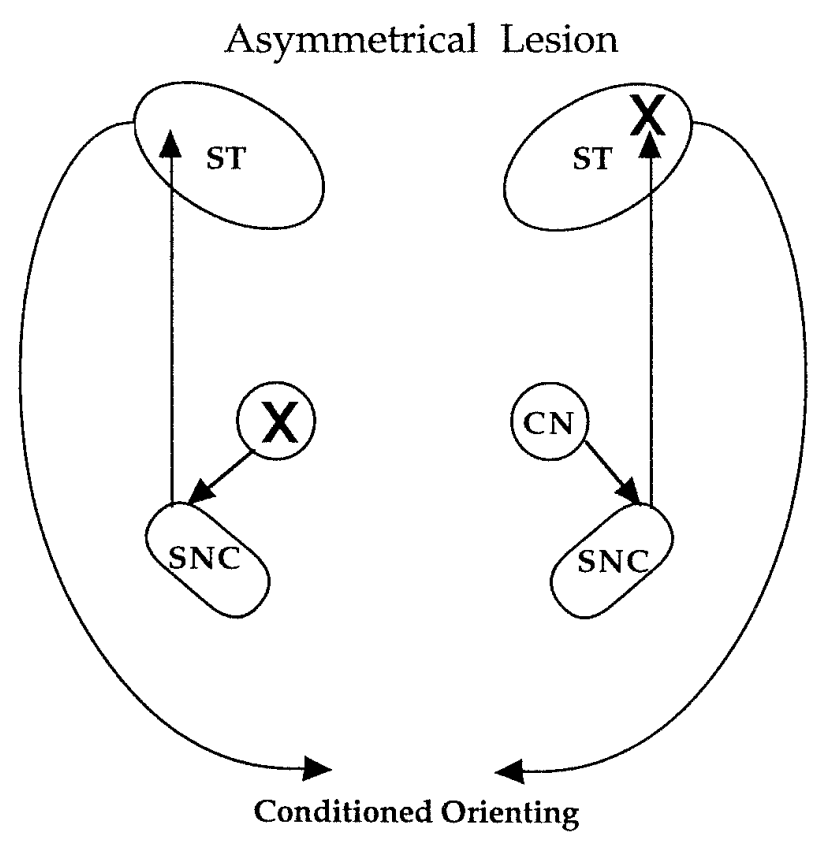

Figure 1. The combination of a unilateral lesion of $\mathrm{CN}$ in one hemisphere (indicated by the $X$ on the top right in the schematic) with a contralateral lesion of the dorsolateral striatum ( $X$ on the lower left) prevents output dependent on the amygdalo-nigrostriatal circuitry shown in the schematic.

hemisphere (Gonzales and Chesselet, 1990), a CN lesion in one hemisphere combined with a 6-hydroxydopamine (6-OHDA) lesion of the dorsolateral striatum on the other side of the brain would effectively eliminate $\mathrm{CN}$ regulation of striatal function. This asymmetrical lesion preparation was used to assess whether conditioned orienting depends on $\mathrm{CN}$ regulation of the ascending nigrostriatal system. If so, the asymmetrical lesion, like bilateral lesions of the amygdala $\mathrm{CN}$, should abolish the acquisition of conditioned orienting responses.

\section{MATERIALS AND METHODS}

Subjects. For the initial experiment, 46 male Long-Evans rats (Charles River Laboratories, Raleigh, NC) were housed in a climate-controlled vivarium (lights on 7:00 A.M. to 7:00 P.M.).

Surgery. Under anesthesia (Nembutal $50 \mathrm{mg} / \mathrm{kg}$ ), 300-325 gm rats received unilateral lesions of amygdala $\mathrm{CN}$ produced by ibotenic acid (Regis Chemical), unilateral lesions of the ST made with 6-hydroxydopamine (6-OHDA, obtained from RBI), or control surgical procedures for those sites. Injection coordinates are given with reference to bregma according to the Paxinos and Watson (1986) rat brain atlas. The $\mathrm{CN}$ lesion was produced at a single injection site (anterior-posterior, $-2.3 \mathrm{~mm}$; medial-lateral, $\pm 4.2 \mathrm{~mm}$; dorsal-ventral, $-7.7 \mathrm{~mm}$ ). The ST lesion was produced at two injection sites (anterior-posterior, $+2.0 \mathrm{~mm}$ and $+0.5 \mathrm{~mm}$; medial-lateral, $\pm 3.0 \mathrm{~mm}$; dorsal-ventral, $-5.5 \mathrm{~mm}$ ). Ibotenic acid $(10 \mathrm{mg} / \mathrm{ml})$ was dissolved in PBS; 6-OHDA (4 mg/ml) was dissolved in physiological saline. Drug or appropriate vehicle was delivered using a Hamilton microsyringe at a volume of $0.2 \mu 1 /$ site in $\mathrm{CN}$ and $1.0 \mu \mathrm{l} / \mathrm{site}$ in ST. Rats in the asymmetrical lesion group (CN/ST) underwent a $\mathrm{CN}$ lesion in one hemisphere (side counterbalanced within the group) and a lesion of ST in the contralateral hemisphere. Rats in the unilateral lesion groups underwent either a $\mathrm{CN}$ or an ST lesion in one hemisphere and injection of the appropriate vehicle into the unlesioned target on the contralateral side.

Apparatus. The testing apparatus consisted of four individual chambers (Coulbourn Instruments, Allentown, PA), each $27.9 \times 25.4 \times 30.5 \mathrm{~cm}^{3}$. The food-cup was recessed in the center of one end wall $2 \mathrm{~cm}$ above the floor; a $6 \mathrm{~W}$ light, which was the source of the visual conditioned stimulus (CS), was located $19 \mathrm{~cm}$ above the recessed food-cup. A $4 \mathrm{~W}$ house light placed in the center on the top of the chamber and a $25 \mathrm{~W}$ red bulb placed $2.1 \mathrm{~m}$ from the chambers provided continuous dim background illumination. A low-light television camera was placed $2.0 \mathrm{~m}$ from the experimental chambers. Videocassette recorders were programmed to record behaviors that occurred during the $10 \mathrm{sec}$ intervals before, during, and after presentation.

Behavioral procedures. After postoperative recovery for 1 week, rats were gradually reduced to $85 \%$ of their free-feeding body weights and maintained at that weight by receiving a premeasured amount of rat chow given each day in the late afternoon.

Ten deliveries of two $45 \mathrm{mg}$ food pellets (Noyes) were given on a variable-time 2 min schedule in a single session to acclimate rats to food delivery. Next, the occurrence and habituation of orienting behavior before conditioning was examined in $2 \mathrm{~d}$ of sessions consisting of four 10 sec light-only trials, with intertrial intervals that averaged $8 \mathrm{~min}$. Then rats received $10 \mathrm{~d}$ of sessions in which five $10 \mathrm{sec}$ presentations of the light were followed immediately by the food unconditioned stimulus (US), with variable intertrial intervals that averaged $8 \mathrm{~min}$. These procedures support the development of associatively conditioned orienting and foodcup responses that are not observed with unpaired presentations of the CS and US (Gallagher et al., 1990).

All observations were made from videotapes and paced by auditory signals recorded on the tapes. For each rat, observations were made at $1.25 \mathrm{sec}$ intervals during the $5 \mathrm{sec}$ period immediately before CS presentations and during CS presentations. At each observation, one and only one behavior was recorded. Two broad categories of behavior are reported. Rear behavior (standing on the hind legs with both front legs off the floor, but not grooming) occurs initially as an unconditioned orienting response to visual CSs, similar to those used in these experiments, but it is also acquired by pairings of the CS with the unconditioned stimulus. Food-cup behavior, the unconditioned behavior (short, rapid horizontal, and/or vertical movements of the head oriented toward the food-cup), initially occurs in response to delivery of the food US, but is rapidly acquired to CSs paired with food. Because previous data (Holland, 1977, 1984) showed that rear behavior occurs primarily during the first $5 \mathrm{sec}$ period of a $10 \mathrm{sec}$ CS, and food-cup behavior occurs primarily during the last $5 \mathrm{sec}$ of the CS, we report the frequencies of rear behavior during the first half of the $10 \mathrm{sec}$ CS intervals and the frequencies of food-cup behavior during the last half of the CS intervals. It is worth noting that because these two behaviors occur primarily at different times, there is little competition between them in performance.

The index of behavioral frequency used was percentage total behavior, obtained by dividing the frequency of the target behavior in any observation interval by the total number of observations made in that interval. Note that because the number of observations was constant within each observation interval, this measure is an absolute frequency measure, not a relative one.

Histology. After completion of behavioral testing, the rats were deeply anesthetized with chloral hydrate $(30 \%)$ and perfused transcardially with $0.9 \%$ cold saline. The brains were rapidly removed and cut coronally into two blocks at the optic chiasm. The posterior block was stored in $10 \%$ formalin for 1 week and then sectioned $(50 \mu \mathrm{m})$ coronally on a freezing microtome, mounted on slides, and Nissl-stained to verify the neurotoxic lesion of $\mathrm{CN}$. The anterior block was stored at $-70^{\circ} \mathrm{C}$ until processing. For autoradiography, coronal sections $(15 \mu \mathrm{m})$ were cut in a cryostat at $-15^{\circ} \mathrm{C}$ and thaw-mounted onto gelatin-coated slides. The slides were refrigerated for $2 \mathrm{hr}$ in a vacuum-sealed desiccator and then stored at $-70^{\circ} \mathrm{C}$ until assays were performed. To define the lesion produced by 6-OHDA, autoradiograms were generated from sections through the striatum using $15 \mathrm{~nm}\left[{ }^{3} \mathrm{H}\right]$ Mazindol (15 Ci/nmol, New England Nuclear), a ligand for DA uptake sites. Methods were identical to those described in a previous published report from this lab (Burwell and Gallagher, 1993). Sections for total binding were done in the presence of $300 \mathrm{~nm}$ desipramine, which selectively inhibits Mazindol binding to norepinephrine uptake sites. To define density of $\left[{ }^{3} \mathrm{H}\right]$ Mazindol labeling of DA uptake sites, nonspecific binding was defined by incubation of adjacent sections in the presence of $30 \mu \mathrm{M}$ benztropine. Subtraction of binding in the presence of $30 \mu \mathrm{M}$ benztropine from total $\left[{ }^{3} \mathrm{H}\right]$ Mazindol binding provided a measure of DA innervation of the dorsolateral striatum in control and ST lesion brains. Densitometry was done on an Imaging system (MCID, St. Catherine, Ontario, Canada) using tritium standards (Amersham). 

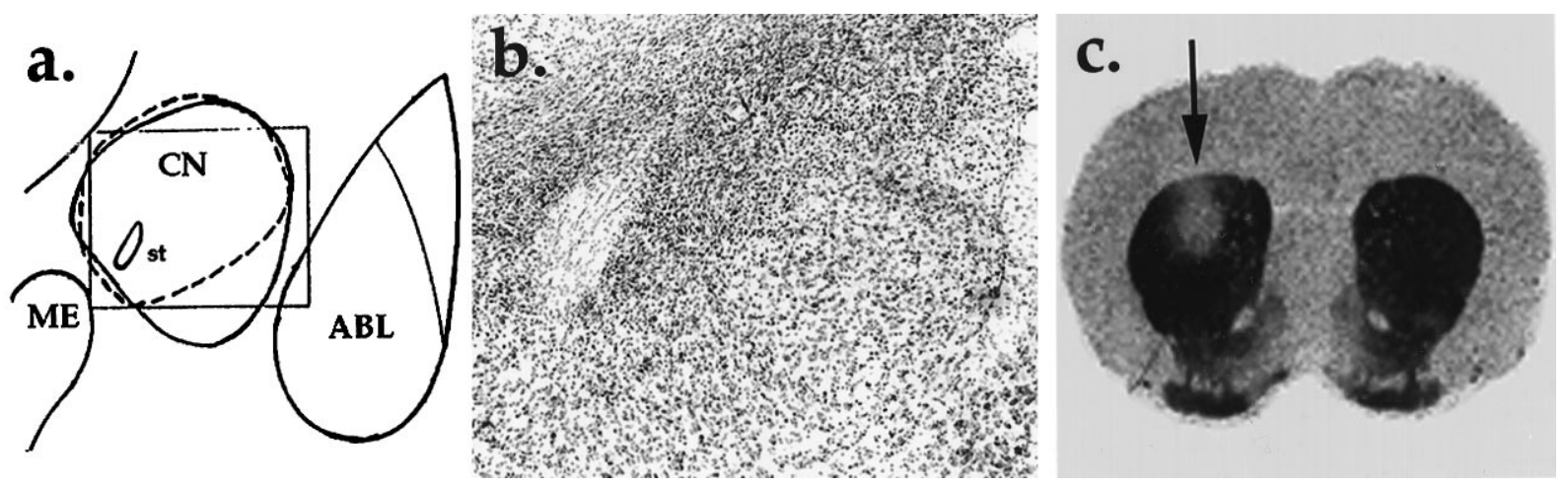

Figure 2. $a$, Diagram of the amygdala complex with the position of the photomicrograph (shown in $b$ ) demarcated by the enclosed box and the area of the lesion identified by broken lines. $b$, Heavy gliosis marks the site of the lesion in the $\mathrm{CN}$ surrounding the stria terminalis. $c$, Autoradiogram shows DA uptake sites labeled with $\left[{ }^{3} \mathrm{H}\right]$ Mazindol. High levels of binding (dark area) are evident throughout the intact striatum on the right. The site of 6-OHDA administration on the left exhibits low binding, indicative of a loss of DA innervation. Average lesion area was $3.03 \mathrm{~mm}^{2}$, and radioactive density averaged $1.88 \mathrm{nCi} / \mathrm{mg}$ relative to $8.35 \mathrm{nCi} / \mathrm{mg}$ on the contralateral side. $A B L$, Basolateral nucleus; $C N$, central nucleus; $M E$, medial nucleus; st, stria terminalis.

\section{Orienting and Habituation}

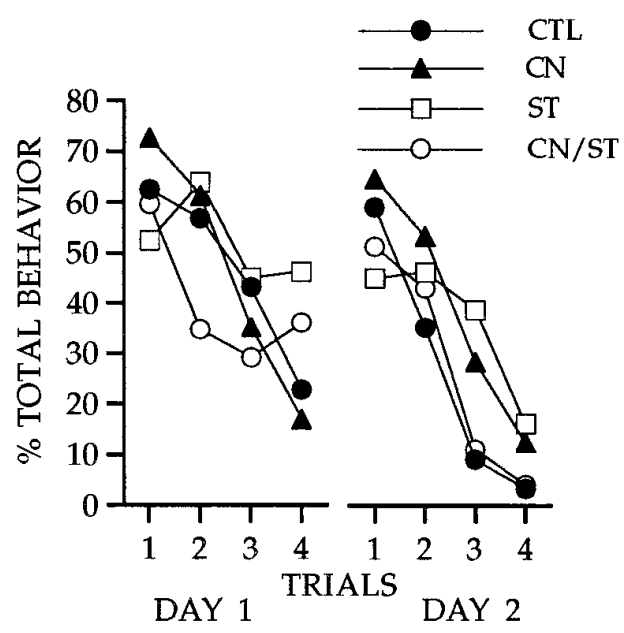

Figure 3. Responses to the visual cue during two sessions of light-alone presentations. All groups showed comparable orienting and habituation during these trials.

\section{RESULTS AND DISCUSSION}

\section{Histology}

Histological analysis revealed that unilateral neurotoxic lesions of $\mathrm{CN}$ were appropriately placed in 9 rats in the asymmetrical $(\mathrm{CN} / \mathrm{ST})$ group and 10 rats in the $\mathrm{CN}$ group. The $\mathrm{CN}$ lesions ranged in size from 30 to $100 \%$, with the average lesion encompassing approximately $55 \%$ of the nucleus, and medial $\mathrm{CN}$ was damaged in all cases. The placement and size of the 6-OHDA lesion of dorsolateral striatum was also comparable between the asymmetrical group and the unilateral ST group. See Figure 2 for representative lesions.

\section{Behavioral data}

Figure 3 shows the occurrence of unconditioned orienting responses to the visual cue. All groups oriented to the light at relatively high levels initially, and comparable habituation was observed for all groups both within and across sessions of lightalone presentations. Although unilateral ST damage typically produces contralateral neglect, a syndrome in which behavioral responses directed contralateral to the lesion are impaired (Carli et al., 1985, 1989; Fairley and Marshall, 1986), the presentation of diffuse illumination as the visual stimulus in the present study elicited an orienting behavior that was not constrained by the location of the stimulus or the direction of the response.

The primary behavioral data for this study are shown in Figure 4. Statistical analysis indicated that the conditioned OR (shown in Fig. 4a) was increased in all groups over the course of training (Friedman test, $\chi^{2}(4) \geq 13.8, p<0.01$ ), except in the asymmetrical lesion group. Responses in the latter group did not change significantly (Friedman test, $\chi^{2}(4)=2.31$, not significant). Independent tests (Mann-Whitney, two-tailed) showed that the asymmetrical lesion group differed from each of the other groups $(p<$ $0.05)$, which did not differ from one another. Statistical analysis of the data in Figure $4 b$ showed that conditioned food-cup responses were not reliably affected by any lesion treatment. Rats in all groups showed increased conditioned food-cup responses over sessions (Friedman test, $\chi^{2}(4) \geq 31.8, p<0.01$ ), and there was no significant difference among the groups (Kruskal-Wallis, $\chi^{2}(3)<$ 5.19, not significant).

Rats with the asymmetrical (CN/ST) lesion failed to acquire the conditioned orienting response, but readily acquired conditioned food-cup behavior. The selective impairment of conditioned orienting was not because of an inability to integrate sensory information with performance of the orienting response; rats with the CN/ST lesion showed normal spontaneous orienting. The behavioral profile of rats in the asymmetrical lesion group resembles that of rats with bilateral $\mathrm{CN}$ lesions described in previous studies (Gallagher et al., 1990). These results are consistent with the interpretation that conditioned orienting depends on amgydalonigrostriatal circuitry.

\section{Experiment 2}

Experiment 2 was conducted to examine the effect of reversible inactivation of the dorsolateral striatum during conditioning. Lidocaine infusion into the dorsolateral striatum was made unilaterally immediately before conditioning sessions, similar to those used in experiment 1 . Rats were monitored during these and subsequent sessions when no lidocaine infusion was made to assess whether intact function of the dorsolateral striatum is essential for the development of conditioned orienting behavior.

\section{MATERIALS AND METHODS}

Subjects. Male Long-Evans rats $(n=32)$ were obtained from Charles River Laboratories (Raleigh, NC) and housed in the same manner as in 
a. Conditioned OR

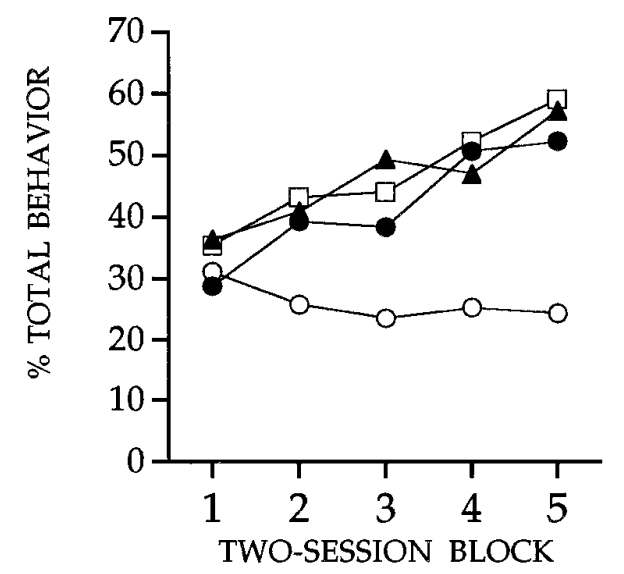

b. Conditioned Food-Cup

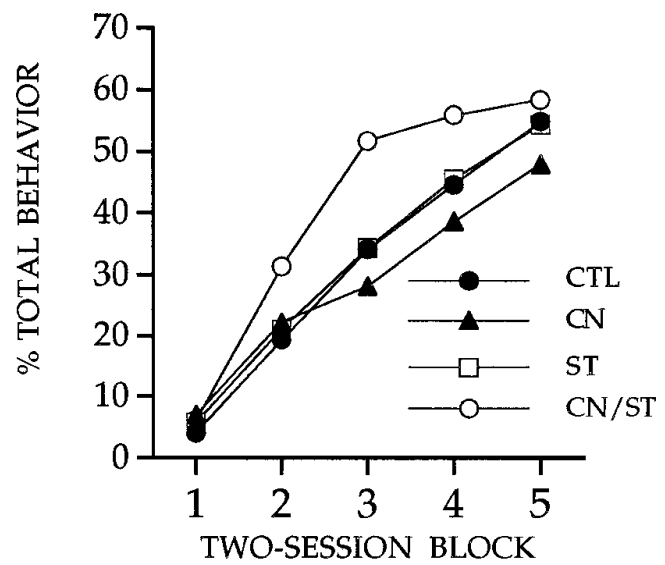

Figure 4. Conditioned orienting behavior $(a)$ and conditioned food-cup behavior $(b)$ during 10 sessions in which the light CS was paired with the food US. Rats with the asymmetrical lesion (group $\mathrm{CN} / \mathrm{ST}$ ) failed to acquire conditioned orienting behavior relative to the acquisition displayed by all other comparison groups. All groups showed comparable acquisition of the conditioned food-cup response. lesion (right). The top right shows gliosis in the CN. Myelin staining at the lesion site, however, is intact (bottom right). Histological analysis revealed that unilateral neurotoxic lesions of $\mathrm{CN}$ were appropriately placed in 10 rats in the symmetrical CN/ST lidocaine group (SYM-paired), 11 rats in the asymmetrical CN/ST lidocaine-paired group (ASYM-paired), and 9 rats in the asymmetrical CN/ST lidocaine-unpaired group (ASYM-unpaired). The $\mathrm{CN}$ lesions ranged in size from 35 to $100 \%$, with the average lesion encompassing approximately $60 \%$ of the nucleus. In all cases, the medial $\mathrm{CN}$ was damaged. Injection sites were confirmed in the dorsolateral striatum. Placements had a similar distribution across the three groups of rats with a cannula implant.

\section{Behavioral results}

Comparable spontaneous orienting and habituation were evident in all groups of rats (Fig. 6). The primary data of interest in this experiment are shown in Figure $7 a$. The occurrence of conditioned orienting to the light differed for the unpaired group and the group with symmetrical CN/ST lidocaine treatment (MannWhitney $\mathrm{U}$ test, $p<0.05)$. In contrast, the rats with asymmetrical $\mathrm{CN} / \mathrm{ST}$ lidocaine did not differ from the unpaired group during the sessions of training under lidocaine treatment. Note, however, that conditioned orienting was evident in rats with asymmetrical $\mathrm{CN} / \mathrm{ST}$ treatment when lidocaine infusion was terminated (Friedman test, $\chi^{2}(1) \geq 7.36, p<0.01$ comparing block 5 with block 4 ) but did not increase further with subsequent training (Friedman test, $\chi^{2}(2)=0.17$, not significant), indicating that dorsal striatum is essential for the expression of the conditioned orienting response. These results further support the conclusion of experiment 1 that conditioned orienting depends on an amygdalonigrostriatal pathway. Also in agreement with the results of experiment 1, inactivation did not affect the development of another conditioned response that was concurrently monitored. Whereas unpaired presentations of the CS and US did not support the development of conditioned food-cup responses (Friedman test, $\chi^{2}(6)=3.48$, not significant), this conditioned behavior emerged equivalently in the groups that received paired CS/US trials (Friedman test, $\chi^{2}(6) \geq 48.3, p<0.01$; see Figure $7 b$ ), regardless of lidocaine treatment.

The results of this experiment are consistent with the interpretation that the dorsolateral striatum is essential for the expression of the conditioned orienting response. During inactivation sessions, the occurrence of this behavior in the ASYM-paired group did not differ from the unpaired group. The results also indicate

\section{The photomicrographs displayed in Figure 5 show Nissl- and
myelin-stained sections for a control rat (left) and a rat with a CN \\ RESULTS AND DISCUSSION \\ Histology}



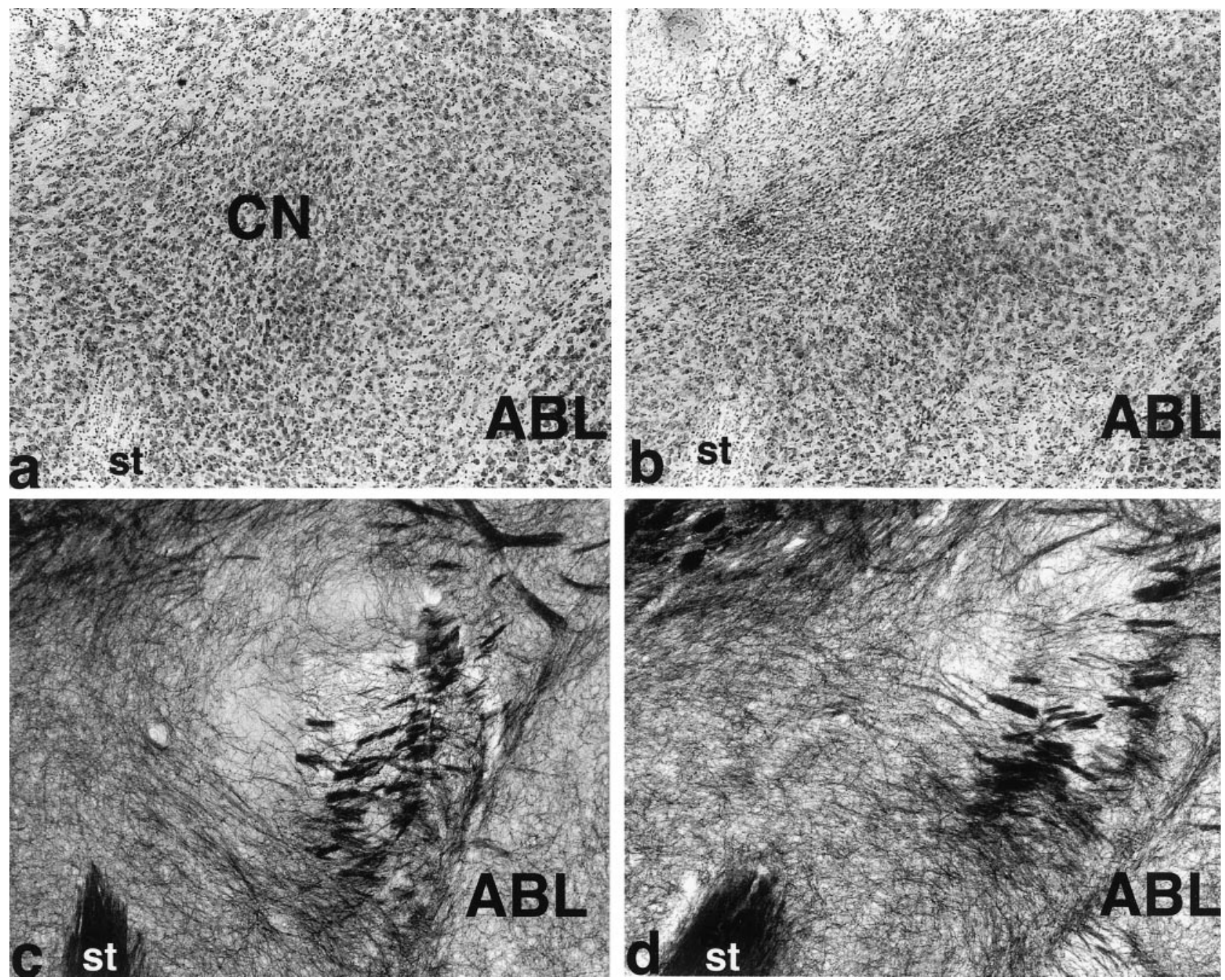

Figure 5. Photomicrographs show Nissl-stained sections (top) and gold chloride myelin-stained sections (bottom) taken in the coronal plane through the amygdala central nucleus. $a$ and $c$ are from a control brain. The neurotoxic lesion brain in $b$ shows heavy gliosis in the $\mathrm{CN}$. Spared neurons are evident in the far lateral $\mathrm{CN}$. However, $d$ shows intact myelin in the lesion area (shown in $b$ ). $A B L$, Basolateral nucleus; $C N$, central nucleus; st, stria terminalis.

that the intact function of the dorsolateral striatum is not essential for the acquisition of the conditioned orienting response, which appeared in the ASYM-paired group when lidocaine infusion was terminated. Because this response in the ASYM-paired group did not achieve the same magnitude as that observed in the SYMpaired group, the role of the striatum and its dopamine innervation may not be entirely limited to the expression of the conditioned response but may also modulate this form of learning.

\section{General Discussion}

Much research has shown that targets of $\mathrm{CN}$ in the brainstem exert control over autonomic and behavioral responses used in the expression of associative learning. Conditioned freezing, heart rate, potentiation of startle, and eyeblink reflexes all appear to depend on output from brainstem systems, which are innervated by CN (Kapp et al., 1984; Hitchcock and Davis, 1991; Whalen and Kapp, 1991; Kim et al., 1993). For example, the acoustic reflex circuit for startle is organized at the level of the brainstem and includes the nucleus pontis caudali (RPC). The CN provides a substantial projection to RPC that is critical for conditioned potentiation of the startle reflex, as demonstrated by the use of

\section{Orienting and Habituation}

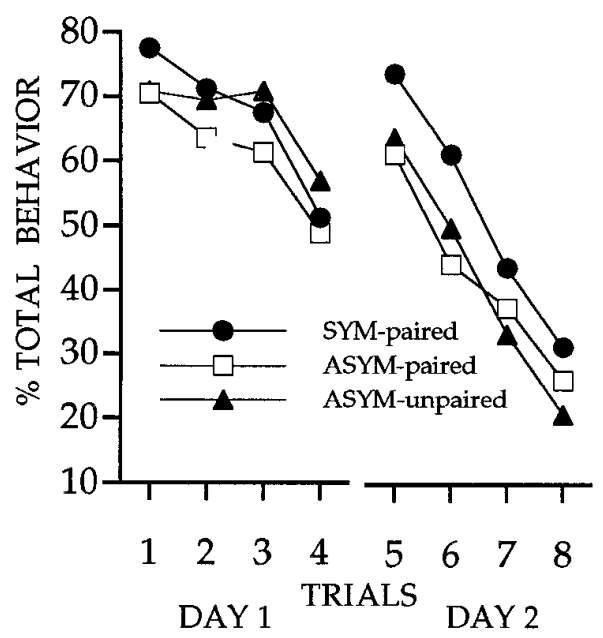

Figure 6. Rats in all groups showed initial orienting and comparable habituation to the visual CS. 


\section{a. Conditioned OR}

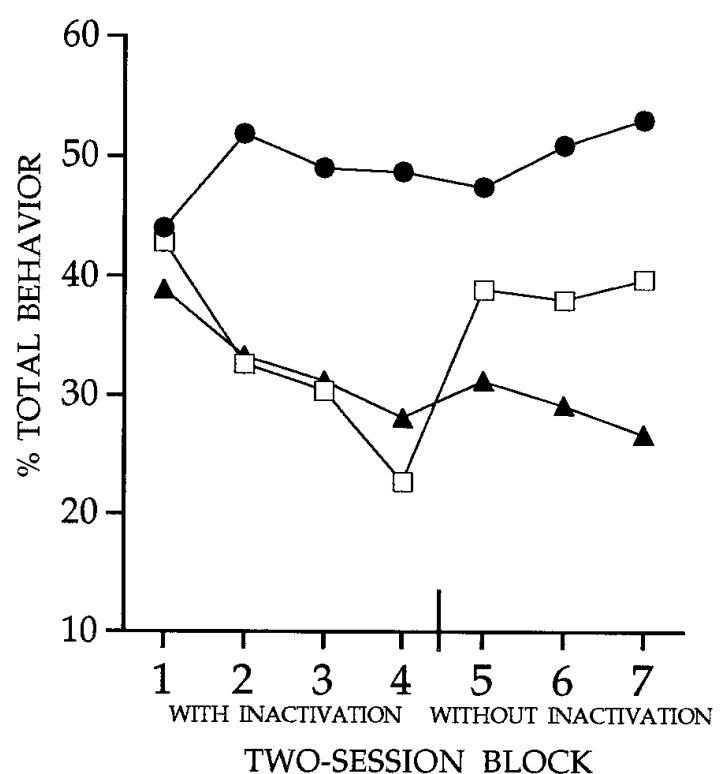

\section{b. Conditioned Food-Cup}

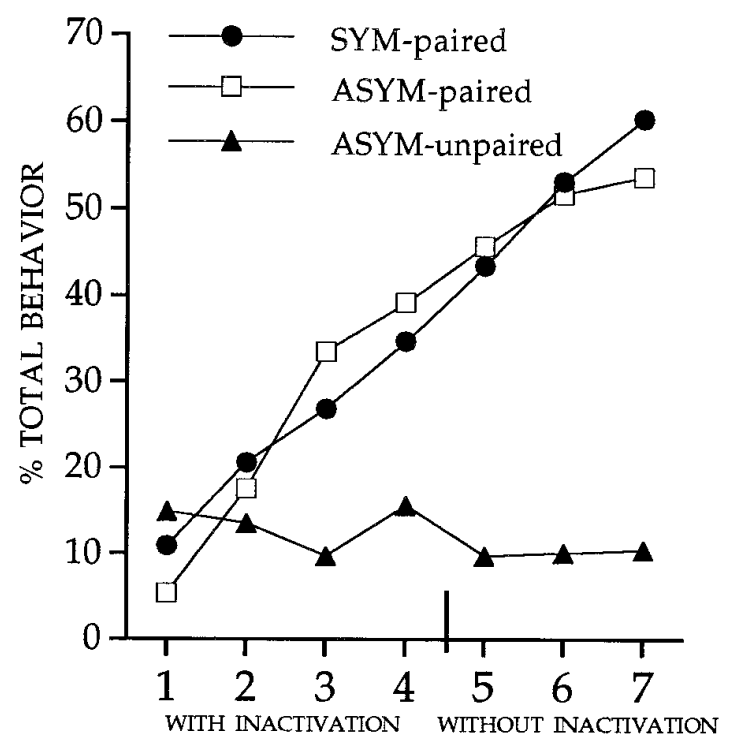

TWO-SESSION BLOCK

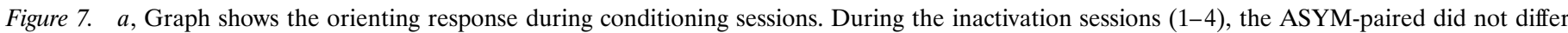

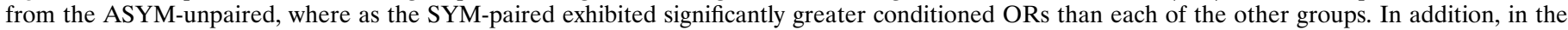



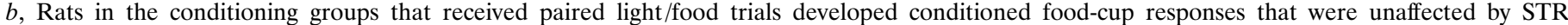
inactivation. Compare SYM-paired and ASYM-paired groups with the ASYM-unpaired group.

asymmetrical lesions (Hitchcock and Davis, 1991). A number of those measures that are amenable to conditioning (e.g., heart rate and startle) resemble orienting responses to novel stimuli. In all cases, $\mathrm{CN}$ lesions that impair conditioning do not affect the normal occurrence or habituation of spontaneous orienting to novel stimuli (Kapp et al., 1979; Gallagher et al., 1980, 1981; Hitchcock and Davis, 1986, 1987). Similarly, the conditioned orienting behavior studied in this research is abolished by bilateral lesions of the $\mathrm{CN}$, which spare unconditioned orienting to novel cues (Gallagher et al., 1990). The results of the current experiments indicate that, along with the direct $\mathrm{CN}$ innervation of brainstem systems that control other CRs, the expression of conditioned orienting appears to be mediated by the $\mathrm{CN}$ through dopamine input to the striatum.

Considerable evidence from other research indicates that the striatum is important for stimulus-response learning and orientation to sensory cues (Carli et al., 1985; Fairley and Marshall, 1989; McDonald and White, 1993). Unilateral damage to the nigrostriatal dopamine system produces a deficit in orienting to stimuli presented contralateral to the site of the lesion, a function that depends on a lateral but not medial region of the striatum (Fairley and Marshall, 1986). Gonzalez and Chesselet (1990) demonstrated that the dorsal tier of dopaminergic neurons in the lateral substantia nigra compacta, which project to the dorsolateral striatum, are innervated by neurons in the medial division of the CN. These amygdala projection neurons are located in a circumscribed anterior and dorsal region of the medial $\mathrm{CN}$, which was the target of the unilateral $\mathrm{CN}$ lesion in the current investigation. Although lesion size within the $\mathrm{CN}$ varied, in all cases substantial damage to the medial $\mathrm{CN}$ at anterior levels was produced. This circumscribed lesion, in combination with an asymmetrical DA lesion or transient inactivation of the dorsolateral striatum, was sufficient to prevent the occurrence of conditioned orienting responses. Thus, the present studies indicate that conditioned orienting behavior depends on connections of the amygdala $\mathrm{CN}$ with the forebrain via the substantia nigra.

In the experiment using transient inactivation of the striatum, orienting responses that were not evident in the ASYM-paired group during inactivation sessions emerged when the inactivation produced by lidocaine infusion was terminated. This result indicates that the intact function of the dorsolateral striatum is not essential for development of this form of associative learning. Rather, the associative basis for conditioned orienting would appear to occur in circuitry afferent to the striatum. This finding is consistent with electrophysiological data indicating that substantia nigra neurons are responsive to conditioned stimuli (Miller et al., 1981; Steinfels et al., 1983; Ljungberg et al., 1992; Mirenowicz and Schultz, 1996) and further suggests that CN innervation of the substantia nigra may contribute to the conditioned neural activity of those neurons. By this view, conditioned neural activity in the nigrostriatal pathway might be eliminated or severely attenuated by lesions of the amygdala $\mathrm{CN}$.

The current work extends the study of neural systems that provide a substrate for simple classical conditioning in the mammalian brain. Although much current research is focused on the study of the amygdala complex in animal models of fear conditioning, the amygdala is involved more broadly in the associative learning through which animals learn that originally neutral cues are predictors of other significant events. This associative function of the amygdala gains widespread access to both brainstem and forebrain circuitry, as indicated by anatomical and functional studies of the projections of the central nucleus. 


\section{REFERENCES}

Burwell RD, Gallagher M (1993) A longitudinal study of reaction time performance in Long-Evans rats. Neurobiol Aging 14:57-64.

Carli M, Evenden JL, Robbins TW (1985) Depletion of unilateral striatal dopamine impairs initiation of contralateral actions and not sensory attention. Nature 313:679-682.

Carli M, Jones GH, Robbins TW (1989) Effects of unilateral dorsal and ventral striatal dopamine depletion on visual neglect in the rat: a neural and behavioral analysis. Neuroscience 29:309-327.

Fairley PC, Marshall JF (1986) Dopamine in the lateral caudateputamen of the rat is essential for somatosensory orientation. Behav Neurosci 100:652-663.

Fallon JH, Moore RY (1990) Catecholamine innervation of the basal forebrain. IV. Topography of the dopamine projection to the basal forebrain and neostriatum. J Comp Neurol 180:545-580.

Gallagher M, Holland PC (1994) The amygdala complex: multiple roles in associative learning and attention. Proc Natl Acad Sci USA 91:11771-11776.

Gallagher M, Kapp BS, Frysinger RC, Rapp PR (1980) Beta-adrenergic manipulation in amygdala central nucleus alters rabbit heart rate conditioning. Pharmacol Biochem Behav 12:419-426.

Gallagher M, Kapp BS, McNall CL, Pascoe JP (1981) Opiate effects in the amygdala central nucleus on heart rate conditioning in rabbits. Pharmacol Biochem Behav 14:497-505.

Gallagher M, Graham PW, Holland PC (1990) The amygdala central nucleus and appetitive Pavlovian conditioning: lesions impair one class of conditioned behavior. J Neurosci 10:1906-1911.

Gonzales C, Chesselet MF (1990) Amygdalonigral pathway: an anterograde study in the rat with Phaseolus vulgaris leucoagglutinin (PHA-L). J Comp Neurol 297:182-200.

Hitchcock JM, Davis M (1986) Lesions of the amygdala, but not of the cerebellum or red nucleus, block conditioned fear as measured with the potentiated startle paradigm. Behav Neurosci 100:11-22.

Hitchcock JM, Davis M (1987) Fear-potentiated startle using an auditory conditioned stimulus: effects of lesions of the amygdala. Physiol Behav 39:403-408.

Hitchcock JM, Davis M (1991) Efferent pathway of the amygdala involved in conditioned fear as measured with the fear-potentiated startle paradigm. Behav Neurosci 105:826-842.

Holland PC (1977) Conditioned stimulus as a determinant of the form of the Pavlovian conditioned response. J Exp Psychol Anim Behav Process 3:77-104.
Holland PC (1984) The origins of Pavlovian conditioned behavior. In: The psychology of learning and motivation, Vol 18 (Bower G, ed), pp 129-173. Englewood Cliffs, NJ: Prentice-Hall.

Kapp BS, Frysinger RC, Gallagher M, Haselton JB (1979) Amygdala central nucleus lesion: effects on heart rate conditioning in the rabbit. Physiol Behav 23:1109-1117.

Kapp BS, Pascoe JP, Bixler MA (1984) The amygdala: a neuroanatomical systems approach to its contribution to aversive conditioning. In: Neuropsychology of memory (Squire LR, Butters N, eds), pp 473-488. New York: Guilford.

Kim JJ, Rison RA, Fanselow MS (1993) Effects of amygdala, hippocampus and periaqueductal gray lesions on short- and long-term context fear. Behav Neurosci 107:1093-1098.

Ljungberg T, Ungerstedt U (1976) Sensory inattention produced by 6-hydroxydopamine-induced degeneration of ascending dopamine neurons in the brain. Exp Neurol 53:585-600.

Ljungberg T, Apicella P, Schultz W (1992) Responses of monkey midbrain dopamine neurons during learning of behavioral reactions. J Neurophysiol 67:145-163.

McDonald RJ, White NM (1993) A triple dissociation of memory systems: hippocampus, amygdala, and dorsal striatum. Behav Neurosci 107:3-22.

Miller JD, Sanghera MK, German DC (1981) Mesencephalic dopaminergic unit activity in the behaviorally conditioned rat. Life Sci 29:1255-1263.

Mirenowicz J, Schultz W (1996) Preferential activation of midbrain dopamine neurons by appetitive rather than aversive stimuli. Nature 379:449-451.

Paxinos G, Watson C (1986) The rat brain in stereotaxic coordinates. New York: Academic.

Sananes CB, Campbell BA (1989) Role of the central nucleus of the amygdala in olfactory heart rate conditioning. Behav Neurosci 103:519-525.

Schmued LC (1990) A rapid, sensitive histochemical stain for myelin in frozen brain sections. J Histochem Cytochem 38:717-720.

Steinfels GF, Heym J, Strecker RE, Jacobs BL (1983) Behavioral correlates of dopaminergic unit activity in freely moving cats. Brain Res 258:217-228.

Whalen PJ, Kapp BS (1991) Contributions of the amygdaloid central nucleus to the modulation of the nictitating membrane reflex in the rabbit. Behav Neurosci 105:141-153. 